\title{
A Survey on Neuropsychological State, Oxidative Stress and Hematological Parameters in Coke Oven Workers
}

\author{
Behnaz Salehiforouz' ${ }^{1}$ Ali Akbar Malekirad1,2*, Mohammad Abdollahi'2, Kobra Rahzani \\ Sara Mostafalou ${ }^{2}$ \\ ${ }^{1}$ Biology Department, Payame Noor University, Tehran, Iran \\ ${ }^{2}$ Faculty of Pharmacy, Pharmaceutical Sciences Research Center, Tehran University of Medical Science, Tehran, \\ Iran \\ ${ }^{3}$ Faculty of Nursing and Midwifery, Arak University of Medical Science, Arak, Iran \\ Email: ${ }^{*}$ AK malekirad@yahoo.com, ${ }^{*}$ Malekirad1973@gmail.com
}

Received 4 April 2014; revised 8 May 2014; accepted 15 May 2014

Copyright (C) 2014 by authors and Scientific Research Publishing Inc.

This work is licensed under the Creative Commons Attribution International License (CC BY). http://creativecommons.org/licenses/by/4.0/

(c) (i) Open Access

\section{Abstract}

Coke oven factories are one of the main emitting sources of polycyclic aromatic hydrocarbons (PAH)s which have been identified as highly carcinogenic chemicals by IARC. Induction of oxidative stress and following disrupting pathways has been repeatedly reported in subjects exposed to PAHs. This study was performed to monitor the oxidative stress markers, hematological and biochemical parameters, and psychological states in coke oven workers. 100 male subjects were included in this comparative cross-sectional study. At first 50 male workers who were 22 - 63 and worked in the steel factory located in South-East of Iran were contained. Control subjects were chosen from Esfahan, in the factory's neighborhood, consisting of 50 office workers without any background of occupational exposure to metals. The blood levels of hematological factors and oxidative stress were measured. In order to compare data, two-sample t test was applied. In order to measure the correlation between variables, Pearson correlation coefficient was applied. The mean level of total antioxidant capacity, hemoglobin $(\mathrm{Hb})$, mean corpuscular hemoglobin concentration (MCHC), and hematocrit were significantly lower in the workers in association with increased lipid per oxidation (LPO). A positive correlation existed between work history, hyper thyroids $\left(r^{2}=0.288, p=0.01\right)$, and endocrine disorders $\left(r^{2}=0.309, p=0.028\right)$. Occupational exposure of coke oven workers to PAHs can induce oxidative stress and consequent metabolic and neuropsychological disorders. When workers take daily shower and use protective tools, absorption of toxic elements would decrease so that reaching them to the body can be suitably limited.

\footnotetext{
${ }^{*}$ Corresponding author.
} 


\section{Keywords}

\section{Coke Oven Workers, Neuropsychological State, Oxidative Stress, Hematological Parameters}

\section{Introduction}

International Agency for Research on Cancer (IARC) has assorted the long-term exposure to coke in workplaces as human carcinogen mostly because of the release of polycyclic aromatic hydrocarbons (PAH) during pyrolys [1]. Coke oven factories have been known to be the main source of emitting PAHs which can be exhaled by people working in such places [2]. Because of frequent exposure to coke oven emissions (COE), workers of coke oven factories are at the risk of developing lung diseases [3]. The adjusted Ors of abnormal ALT and AST was seen among those workers who had higher COE exposure. It has also been shown that the risks of abnormal ALT due to COE exposure are more raised in overweight people (body mass index (BMI) $>$ or $=25 \mathrm{~kg} / \mathrm{m}$ (2)) [4].

Immunological changes in association with oxidative stress and lipid peroxidation have been reported for coke oven workers who are chronically exposed to PAHs [5].

Elevated internal PAH burden due to COE exposure can cause disruption of redox system, oxidative stress, and genetic damages [6]. Usually when oxidant elements defeat body antioxidants, lipid peroxidation, oxidative protein modification, and DNA damage can occur in association with other disrupting signals leading to a lot of health disorders [7].

In order to maintain the level of oxidant state under control, living organisms have advanced their antioxidant systems composed of non-enzymatic agents like glutathione, ascorbic acid, tocopherol, carotene, uric acid, and bilirubin and enzymatic scavengers such as superoxide dismutase (SOD), glutathione peroxidase (GPx), and catalase (CAT) [7]-[9]. In this regard, measuring total antioxidant capacity (TAC) of biological systems has this value to evaluate the overall effect of a chemical on the interactions between oxidative and anti-oxidative elements in the redox system.

In our previous studies, it was declared that being exposed to industrial pollution can result in modified oxidative toxicity in exposed workers [10]-[12]. So the present research was performed to consider the oxidative stress, its clinical signs, and neurocognitive impairments in coke oven workers.

\section{Materials and Methods}

Tetraethoxypropane, 2-thiobarbituric acid (TBA), trichloroacetic acid (TCA), n-butanol 2,4,6-tripyridyl-striazine (TPTZ) from Sigma-Aldrich Chemie (Gmbh Munich, Germany), $\mathrm{HNO}_{3}, \mathrm{H}_{2} \mathrm{SO}_{4}, \mathrm{HCL}, \mathrm{NaCl}, \mathrm{PdCl}_{2}, \mathrm{Cu}$ $\left(\mathrm{NO}_{3}\right)_{2}-3 \mathrm{H}_{2} \mathrm{O}$ from Merck Chemical Co. (Tehran, Iran) were applied.

\section{Study Population}

A comparative research was conducted by 100 male subjects in 2012. Before the beginning the research, all subjects expressed their informed satisfaction. The research was approved in Ethics Review Board of Pharmaceutical Sciences Research Center, Tehran, Iran. At first 50 male workers who were 22 to 63 and worked in a steel factory located in South-East of Iran were included. The factory has been working since 1967 and produced 106,000 tons per year. Control subjects were selected from Esfahan, in factory's neighborhood, consisting of 50 office workers, without any experience in jobs related to contact to metals.

By administrating an interview, some information such as work history, socioeconomic states i.e. income and education, and habits like smoking, drug use, consumption of vitamin or antioxidant supplements, drinking alcohol, and routine dietary were elicited by a questionnaire. Those subjects experienced antioxidant consumption, alcohol use, chemo- or radiotherapy, exposure to other toxic materials or substance abuse were excluded from the research. Then, every participant took part in a separate interview. As a rule, all participants should complete clinical assessment to exclude those having any symptoms of chronic diseases like cancer, thyroid disturbance, diabetes, arterial hypertension and anemia. Before going to the workplace, blood samples were taken from participants between 7 and 8 a.m. when worker go to the work at the beginning of the week on Saturdays in Iran.

In order to analyze oxidative stress and hematological markers, blood samples were gathered into heparinized 
tubes and were centrifuged at $3000 \mathrm{~g}$ for 5 min without delay and the plasma was separated and freezed at $-80^{\circ} \mathrm{C}$

\section{Determination of Oxidative Stress Markers}

Thiobarbitturic acid reactive substances (TBARS) method was practiced to determine lipid peroxidation (LPO). Different aldehydes like malondialdehyde (MDA) are produced from oxidative processes of lipids and can react with TBA in acidic conditions and high temperatures due to free radicals attack. The obtained compound has greatest absorbance at $532 \mathrm{~nm}$.

Total antioxidant capacity (TAC) was also measured based on the force of plasma in decreasing $\mathrm{Fe}^{3+}$ to $\mathrm{Fe}^{2+}$ in attendance of TPTZ. The produced blue complex, $\mathrm{Fe}^{2+}-\mathrm{TPTZ}$, has a maximum absorbance in $593 \mathrm{~nm}$. More details of measurement of both LPO and TAC have been explained in our previous paper [9].

\section{To Assess Affective Mood State Fluctuation}

The Iranian version of Profile of Mood States (POMS) assessment has been proven itself to be an excellent measure of affective mood state fluctuation in a wide variety of populations including psychiatric outpatients, medical patients, and in sports psychology. The POMS assesses transient and fluctuating affective mood states. The POMS measures six identifiable mood or affective states: 1) Tension-Anxiety, 2) Vigour-Activity, 3) Depression-Dejection, 4) Fatigue-Inertia, 5) Anger-Hostility, 6) Confusion-Bewilderment [13].

\section{Statistical Analysis}

Two-sample t test was applied in order to compare data. After planning and testing equal variances, multivariate analysis variance was practiced for statistical comparisons. Pearson correlation coefficient employed to establish the relation between variables. Data have been shown regarding their mean \pm SD. So, p values less than 0.05 were considered statistically meaningful. Stats Direct 2.7.9 was used to analyze the data.

\section{Results}

The mid-levels of participant's age, smoking habits, duration of employment, (years \& day), and sex were indicated in Table 1. No statistically meaningful difference was seen between two workers and control groups based on their age, smoking habits and experience for work. The cases mentioned in Table 2 were the mid-levels of fasting blood glucose (FBS), blood urea nitrogen (BUN), aspartate aminotransferase (AST) and alanine aminotransferase (ALT), alkaline phosphates (ALP), hemoglobin (Hb), white blood cells (WBC), red blood cells (RBC), hematocrit, LPO, and TAC, mean corpuscular hemoglobin concentration (MCHC), mean corpuscular hemoglobin (MCH), triglycerides (TG), red blood cell distribution width (RDW), platelets, cholesterol (Cl), cratinine, high-density lipoprotein (HDL), low-density lipoprotein (LDL), mean corpuscular volume (MCV). The mean level of TAC, Hb hematocrit and MCHC were significantly reduced in the workers, whereas LPO increased. The mean of anger, depression, fatigue, friend, confusion, tension in both experimental and control groups are presented in table. The mean of anger, depression, and fatigue were significantly higher whereas vigor and friendliness were lower in the workers in comparison with controls (Table 3). On the other hand, a positive correlation was found between work history, hyper thyroids $\left(r^{2}=0.288, p=0.01\right)$ and endocrine disorders $\left(r^{2}=0.309, p=0.028\right)$.

\section{Discussion}

Making a comparison between controls and workers in this study, the workers have declared significantly higher neuropsychological distress in association with a decrease in LPO and an increase in TAC, MCHC, Hb, and hematocrit. Existence of a positive correlation between years of working and clinical symptoms also indicate that exposure to coke has led to a sort of toxicities in the workers. Interviews have clarified that most of the workers not only used no suitable protective equipment's like face cover, gloves, head cover, long boots and masks, but also did not take a regular shower, though showers were available for them. Cloth mask was their only safety tool. The results of this research indicate that the rank of TAC reduced and LPO elevated in the workers in comparison with the controls. 
Table 1. Summary of demographic data in workers and controls.

\begin{tabular}{cccc}
\hline Subjects & Workers $(\mathbf{n}=\mathbf{5 0})$ & Controls $(\mathbf{n}=\mathbf{5 0})$ & p value \\
\hline Age (years) & $35 \pm 6.85$ & $35.51 \pm 8.22$ & 0.738 \\
Sex & 50 (male) & 50 (male) & - \\
Work history (years) & $11.18 \pm 6.820$ & $10.16 \pm 8.87$ & 0.62 \\
Smoking (years) & $0.76 \pm 3.305$ & $1.80 \pm 4.90$ & 0.216 \\
Smoking (N/day) & $0.70 \pm 3.284$ & $1.50 \pm 4.53$ & 0.315 \\
BMI & $25.65 \pm 1.94$ & - & - \\
\hline
\end{tabular}

Data represent mean $\pm \mathrm{SD}$.

Table 2. Levels of hematological, biochemical and oxidative stress markers in the subjects.

\begin{tabular}{cccc}
\hline Group & Control & Worker & p value \\
\hline WBC (cells/mcL) & $6.3102 \pm 1.39347$ & $6.6939 \pm 1.40095$ & 0.177 \\
RBC (cells/mcL) & $5.6469 \pm 0.47923$ & $5.5680 \pm 0.60865$ & 0.477 \\
Hemoglobin (g/dl) & $16.2347 \pm 1.23551$ & $15.5204 \pm 1.25333$ & 0.005 \\
Hematocrit (\%) & $48.4980 \pm 3.33532$ & $47.2388 \pm 2.88551$ & 0.048 \\
MCH (pg) & $28.8286 \pm 1.94915$ & $28.0771 \pm 2.79819$ & 0.128 \\
MCHC (\%) & $33.4469 \pm 1.04744$ & $32.8020 \pm 1.57089$ & 0.019 \\
RDW (\%) & $12.8469 \pm 0.83644$ & $12.7898 \pm 1.10816$ & 0.774 \\
Platelets (×109/L) & $222.27 \pm 46.976$ & $229.92 \pm 53.228$ & 0.452 \\
MPV & $10.2776 \pm 0.82696$ & $9.9750 \pm 0.96503$ & 0.1 \\
MCV (fl) & $86.1612 \pm 4.32747$ & $85.4612 \pm 6.26813$ & 0.522 \\
FBS (mg/dl) & $87.73 \pm 12.414$ & $86.74 \pm 12.708$ & 0.695 \\
Triglyceride (mg/dl) & $221.47 \pm 187.948$ & $163.59 \pm 126.667$ & 0.3 \\
Cholesterol (mg/dl) & $191.47 \pm 39.911$ & $174.24 \pm 36.431$ & 0.198 \\
LDL (mg/dl) & $69.3333 \pm 15.27525$ & $85.2500 \pm 30.89094$ & 0.456 \\
HDL (mg/dl) & $44.8000 \pm 5.76194$ & $40.7778 \pm 6.03692$ & 0.249 \\
AST (U/L) & $26.20 \pm 9.834$ & $21.42 \pm 6.331$ & 0.245 \\
ALT (U/L) & $42.20 \pm 29.736$ & $27.17 \pm 17.893$ & 0.213 \\
ALP (IU/L) & $181.60 \pm 32.161$ & $203.22 \pm 47.354$ & 0.384 \\
BUN (mg/dl) & $32.31 \pm 6.462$ & $30.56 \pm 5.211$ & 0.142 \\
Creatinine & $1.1500 \pm 0.07071$ & $1.1000 \pm 0.15275$ & 0.678 \\
TAC (mmol/ml) & $2.4147 \pm 0.43922$ & $1.7856 \pm 0.52221$ & 0.0001 \\
LPO (nmol/ml) & $6.78745 \pm 6.462919$ & $15.32357 \pm 12.231441$ & 0.0001 \\
\hline
\end{tabular}

Data represent mean $\pm \mathrm{SD}$.

Table 3. The status of psychological distress in the subjects.

\begin{tabular}{|c|c|c|c|c|c|}
\hline Group & Control & Worker & p value & DF & $\mathbf{F}$ \\
\hline Anger & $4.15 \pm 4.725$ & $7.88 \pm 6.231$ & 0.001 & 1 & 10.997 \\
\hline Depression & $4.94 \pm 5.571$ & $8.19 \pm 7.98$ & 0.022 & 1 & 5.410 \\
\hline Fatigue & $2.67 \pm 3.44$ & $4.95 \pm 4.461$ & 0.006 & 1 & 7.908 \\
\hline Friend & $17.08 \pm 4.058$ & $14.07 \pm 4.827$ & 0.001 & 1 & 10.885 \\
\hline Confusion & $5.17 \pm 2.299$ & $5.21 \pm 2.833$ & 0.945 & 1 & 0.005 \\
\hline Tension & $8.73 \pm 4.257$ & $10.40 \pm 4.578$ & 0.070 & 1 & 3.361 \\
\hline Vigor & $16.69 \pm 5.327$ & $14.284 \pm 5.474$ & 0.033 & 1 & 4.711 \\
\hline Somatic symptoms & $4.67 \pm 3.148$ & $5.45 \pm 3.556$ & 0.254 & 1 & 1.319 \\
\hline Anxiety \& insomnia & $5.23 \pm 4.052$ & $5.83 \pm 3.875$ & 0.455 & 1 & 0.562 \\
\hline Social dysfunction & $5.94 \pm 1.944$ & $5.64 \pm 2.663$ & 0.512 & 1 & 0.433 \\
\hline Severe depression & $1.88 \pm 2.237$ & $3.94 \pm 3.886$ & 0.002 & 1 & 10.608 \\
\hline GHQ-28 & $17.7308 \pm 8.2983$ & $21.0213 \pm 10.7248$ & 0.089 & 1 & 2.945 \\
\hline
\end{tabular}

Data represent mean \pm SD. 
There are several reports on elevated levels of oxidative stress markers in biological samples taken from coke oven workers. Chen et al. [14] have shown that urinary concentration of MDA in COE-exposed workers was considerably higher than non-exposed workers. Elevated urinary levels of 8-hydroxy-2-deoxyguanosine (8OHdG) and 1-hydroxypyrene (1-OHP) as the valid biomarkers of oxidative DNA damage and PAH exposure, respectively, have also been reported repeatedly in coke oven workers [15]-[17]. Furthermore, Liu et al. [6] [18] have reported that the activity of glutathione-s-transferase (GST) in serum of workers occupationally dealing with coke was higher than non-exposed workers. There are also some reports on the elevated level of polycyclic aromatic hydrocarbon (PAH)-DNA adducts in blood cells of foundry and coke oven workers, workers with high levels of exposure to environmental air pollution, and smokers in association decreased antioxidants in the serum [19]. Apart from the referents, the level of ALT in workers with high exposure was $46 \%$ higher (95\% confidence interval $(\mathrm{CI})=7 \%$ - 98\%) than those with low exposure after adjusting for suitable confounders in multivariate models. High exposed workers had also expressed a mean AST level which was $31 \%$ higher (95\% CI = $9 \%-57 \%$ ) than that of low exposed group. However, the increase of an unusual model for liver enzymes (AST > $37 \mathrm{IU} / \mathrm{L}$ or ALT > $39 \mathrm{IU} / \mathrm{L}$ ) was more ordinary in the high exposure group compared to the low exposure group (adjusted odds ratio $=4.4 ; 95 \% \mathrm{CI}=0.9$ - 22.6). Hence, heavy inhalational exposure to COE can cause high ALT and AST levels among topside coking workers due to aberration of liver function and undesirable hepatic effect seem to be mediated through a combination of hazards rather than a unique recognizable chemical [4] [17]. Hu et al. (2006) has stated that the risk of chronic obstructive pulmonary diseases (COPD) in people with high exposure to COE and cigarette smoking was 58-fold in comparison with non-smokers and those who were not exposed to COE [3]. The outcome of the research shows that psychological disorders were higher in the workers in comparison with control group. In support of these results, Nie et al. (2008) have already reported that the scores of total digital span, the forward digital span, and right dotting in the coke oven workers were lower but that of total dotting was higher with a statistical significance [20]. Findings of another study monitored digital span and order digital span scores showed that memory and learning abilities were considerably reduced in coke oven workers [21]. Also the performances on correct pursuit aiming, simple reaction time, and error pursuit aiming have been shown to decrease in coke oven workers exposed to airborne enzo $[\alpha]$ pyree [22]. It seems that not only many other factors involved in workers' lifestyles which are engaged in neurocognitive impairment, but also unknown roles of the oxidative stress with a highlighted background in damaging different parts of nervous system should not be ignored, particularly in long term exposures.

\section{Conclusion}

Taken together, the results of our study indicate that occupational exposure to COE can disrupt the biochemical homeostasis of the body by affecting the redox state. Induction of oxidative stress has a detrimental role in carcinogenic effect of PAHs which are the main components of COE. So, detecting oxidant biomarkers in coke oven workers can have an alarming message regarding their health state in the long future. Our study had also a new finding on the existence of a link between exposure to $\mathrm{COE}$ and neuro-psychological disorders in coke oven factories. Paying attention to the increasing prevalence of psychological diseases in the societies, this effect can exacerbate the health hazards of occupational exposure to COE and invites environmental health researchers for more considerations. However, it is worth mentioning the importance of applying protective tools and developing efficient measures to limit the exposure level of coke oven workers and their family to COE and related toxic chemicals. Some of limitations in study were the lack of measurement of polycyclic aromatic hydrocarbons and also, the lack of control diet in workers and control group.

\section{Acknowledgements}

All in all, the researchers want to express their utmost appreciation to the nice and friendly assistance of all participants and authorities of the factory. This research was the conclusion of master thesis of the second author at Payame Noor University (PNU) financial support for this effort was provided by the Esfahan Payame Noor University and Mohit Palayesh Arya Scientific Company. Authors thank assistance of Iran National Science Foundation (INSF).

\section{References}

[1] Cavallo, D., Ursini, C.L., Pira, E., Romano, C., Maiello, R., Petyx, M. and Iavicoli, S. (2008) Evaluation of DNA 
Damage Induction on Human Pulmonary Cells Exposed to PAHs from Organic Extract of PM10 Collected in a Coke-Oven Plant. Acta Biomed, 79, 97-103.

[2] Castorena-Torres, F., Bermúdez de León, M., Cisneros, B., Zapata-Pérez, O., Salinas, J.E. and Albores, A. (2008) Changes in Gene Expression Induced by Polycyclic Aromatic Hydrocarbons in the Human Cell Lines HepG2 and A549. Toxicology in Vitro, 22, 411-421. http://dx.doi.org/10.1016/j.tiv.2007.10.009

[3] Hu, Y., Chen, B., Yin, Z., Jia, L., Zhou, Y. and Jin, T. (2006) Increased Risk of Chronic Obstructive Pulmonary Diseases in Coke Oven Workers: Interaction between Occupational Exposure and Smoking. Thorax, 61, 290-295. http://dx.doi.org/10.1136/thx.2005.051524

[4] Hu, Y., Chen, B., Qian, J., Jin, L., Jin, T. and Lu, D. (2010) Occupational Coke Oven Emissions Exposure and Risk of Abnormal Liver Function: Modifications of Body Mass Index and Hepatitis Virus Infection. Occupational and Environmental Medicine, 67, 159-165. http://dx.doi.org/10.1136/oem.2009.047316

[5] Jeng, H.A., Pan, C.H., Diawara, N., Chang-Chien, G.P., Lin, W.Y., Huang, C.T., Ho, C.K. and Wu, M.T. (2011) Polycyclic Aromatic Hydrocarbon-Induced Oxidative Stress and Lipid Peroxidation in Relation to Immunological Alteration. Occupational and Environmental Medicine, 68, 653-658. http://dx.doi.org/10.1136/oem.2010.055020

[6] Liu, A.L., Lu, W.Q., Wang, Z.Z., Chen, W.H., Lu, W.H., Yuan, J., Nan, P.H., Sun, J.Y., Zou, Y.L., Zhou, L.H., Zhang, C. and Wu, T.C. (2006) Elevated Levels of Urinary 8-Hydroxy-2-deoxyguanosine, Lymphocytic Micronuclei, and Serum Glutathione S-Transferase in Workers Exposed to Coke Oven Emissions. Environmental Health Perspectives, 114, 673-677. http://dx.doi.org/10.1289/ehp.8562

[7] Abdollahi, M., Ranjbar, A., Shadnia, S., Nikfar, S. and Rezaie, A. (2004) Pesticides and Oxidative Stress: A Review. Medical Science Monitor, 10, 141-147.

[8] Malekirad, A.A., Ranjbar, A., Rahzani, K., Kadkhodaee, M., Rezaie, A., Taghavi, B. and Abdollahi, M. (2005) Oxidative Stress in Operating Room Personnel: Occupational Exposure to Anesthetic Gases. Human \& Experimental Toxicology, 24, 597-601. http://dx.doi.org/10.1191/0960327105ht565oa

[9] Malekirad, A.A., Ranjbar, A., Rahzani, K., Pilehvarian, A.A., Rezaie, A., Zamani, M.J. and Abdollahi, M. (2005) Oxidative Stress in Radiology Staff. Environmental Toxicology and Pharmacology, 20, 215-218. http://dx.doi.org/10.1016/j.etap.2005.01.005

[10] Malekirad, A.A., Oryan, S., Fani, A., Babapor, V., Hashemi, M., Baeeri, M., Bayrami, Z. and Abdollahi, M. (2010) Study on Clinical and Biochemical Toxicity Biomarkers in a Zinc-Lead Mine Workers. Toxicology and Industrial Health, 26, 331-337. http://dx.doi.org/10.1177/0748233710365697

[11] Malekirad, A.A., Mirabdollahi, M., Pilehvarian, A.A., Nassajpour, A.R. and Abdollahi, M. (2013) Neurocognitive Impairment, Clinical and Biochemical Toxicity Biomarkers in Iron-Steel Workers. Toxicology \& Industrial Health, Epub Ahead of Print.

[12] Fazli, D., Malekirad, A.A., Pilehvarian, A.A., Salehi, H., Zerratpishe, A., Rahzani, K. and Abdollahi, M. (2012) Effects of Melissa officinalis L. on Oxidative Status and Biochemical Parameters in Occupationally Exposed Workers to Aluminum: A before after Clinical Trial. International Journal of Pharmacology, 8, 455-458. http://dx.doi.org/10.3923/ijp.2012.455.458

[13] VaezMosavi, S.M.K. and Samandar, Gh. (2002) Standardization of Profile of Mood States in Elite Sports. Olympic Journal, 4, 5-18.

[14] Chen, J.L., Huang, Y.J., Pan, C.H., Hu, C.W. and Chao, M.R. (2011) Determination of Urinary Malondialdehyde by Isotope Dilution LC-MS/MS with Automated Solid-Phase Extraction: A Cautionary Note on Derivatization Optimization. Free Radical Biology and Medicine, 51, 1823-1829. http://dx.doi.org/10.1016/j.freeradbiomed.2011.08.012

[15] Chen, B., Hu, Y.P., Zheng, L.X., Wang, Q.Y., Zhou, Y.F. and Jin, T.Y. (2005) Comparison on Urinary 1-Hydroxypyrene Concentrations between Coke Oven Workers and Non-Occupational Exposed Individuals. Zhonghua Yu Fang Yi Хue Za Zhi, 39, 388-391.

[16] Hu, C.W., Wu, M.T., Chao, M.R., Pan, C.H., Wang, C.J., Swenberg, J.A. and Wu, K.Y. (2004) Comparison of Analyses of Urinary 8-Hydroxy-2'-deoxyguanosine by Isotope-Dilution Liquid Chromatography with Electrospray Tandem Mass Spectrometry and by Enzyme-Linked Immunosorbent Assay. Rapid Communications in Mass Spectrometry, 18, 505-510. http://dx.doi.org/10.1002/rcm.1367

[17] Wu, M.T., Mao, I.F., Wypij, D., Ho, C.K., Chen, J.R. and Christiani, D.C. (1997) Serum Liver Function Profiles in Coking Workers. American Journal of Industrial Medicine, 32, 478-486.

http://dx.doi.org/10.1002/(SICI)1097-0274(199711)32:5<478::AID-AJIM7>3.0.CO;2-O

[18] Liu, A.L., Zou, Y.L., Lu, W.H., Wang, Z.Z. and Lu, W.Q. (2005) Analysis of Serum Glutathione S-Transferase and Urinary 8-Hydroxy-2-deoxyguanosine in Coke Oven Workers. Zhonghua Lao Dong Wei Sheng Zhi Ye Bing Za Zhi, 23, 343-346.

[19] Santella, R.M. (1997) DNA Damage as an Intermediate Biomarker in Intervention Studies. Proceedings of the Society 
for Experimental Biology and Medicine, 216, 166-171. http://dx.doi.org/10.3181/00379727-216-44166

[20] Nie, J.S., Zhang, H.M., Sun, J.Y., Shi, Y.T., Wang, F., Zeng, P., Wang, L.P., Song, J., Wang, J., Liu, H.J. and Niu, Q. (2008) Characteristic of the Neurobehavioral Functional Changes in Coke Oven Workers. Zhonghua Yu Fang Yi Xue Za Zhi, 42, 25-29.

[21] Niu, Q., Zhang, H., Li, X. and Li, M. (2010) Benzo[a]pyrene-Induced Neurobehavioral Function and Neurotransmitter Alterations in Coke Oven Workers. Occupational and Environmental Medicine, 67, 444-448. http://dx.doi.org/10.1136/oem.2009.047969

[22] Qiu, C., Peng, B., Cheng, S., Xia, Y. and Tu, B. (2013) The Effect of Occupational Exposure to Benzo[a]pyrene on Neurobehavioral Function in Coke Oven Workers. American Journal of Industrial Medicine, 56, 347-355. http://dx.doi.org/10.1002/ajim.22119

\author{
Abbreviation \\ Hemoglobin (Hb) \\ Mean Corpuscular Hemoglobin Concentration (MCHC) \\ Lipid Per Oxidation (LPO) \\ Agency for Research on Cancer (IARC) \\ Polycyclic Aromatic Hydrocarbons (PAH) \\ Coke Oven Emissions (COE) \\ Body Mass Index (BMI) \\ Superoxide Dismutase (SOD) \\ Glutathione Peroxidase (GPx) \\ Catalase (CAT) \\ Total Antioxidant Capacity (TAC) \\ 2-Thiobarbituric Acid (TBA) \\ Trichloroacetic Acid (TCA) \\ 2,4,6-Tripyridyl-Striazine (TPTZ) \\ Thiobarbitturic Acid Reactive Substances (TBARS) \\ Malondialdehyde (MDA) \\ Profile of Mood States (POMS) \\ Fasting Blood Glucose (FBS) \\ Blood Urea Nitrogen (BUN) \\ Aspartate Aminotransferase (AST) \\ Alanine Aminotransferase (ALT) \\ Alkaline Phosphates (ALP) \\ Hemoglobin $(\mathrm{Hb})$ \\ White Blood Cells (WBC) \\ Red Blood Cells (RBC) \\ Mean Corpuscular Hemoglobin Concentration (MCHC) \\ Mean Corpuscular Hemoglobin (MCH) \\ Triglycerides (TG) \\ Red Blood Cell Distribution Width (RDW) \\ Cholesterol (Cl) \\ High-Density Lipoprotein (HDL) \\ Low-Density Lipoprotein (LDL) \\ Mean Corpuscular Volume (MCV) \\ 8-Hydroxy-2-Deoxyguanosine (8OHdG) \\ 1-Hydroxypyrene (1-OHP) \\ Glutathione-S-Transferase (GST) \\ Chronic Obstructive Pulmonary Diseases (COPD)
}

